Research Article

\title{
Kinematic Modeling and Simulation of Dual-Arm Robot
}

\author{
Jiwu Wang, Junxiang $\mathrm{Xu}^{*}$ \\ School of Mechanical and Electronic Engineering, Beijing Jiaotong University, Beijing, Haidian District, China
}

\section{ARTICLE INFO}

\section{Article History}

Received 02 December 2020

Accepted 22 April 2021

\section{Keywords}

Dual-arm robots

real-time

inverse kinematics

simulation

\begin{abstract}
In recent years, dual-arm robots have attracted more and more attention due to their advantages such as strong cooperation ability and high flexibility. With the improvement of real-time requirement of dual-arm cooperation, the inverse kinematics solution of robot becomes a key problem to be solved urgently. To solve the time-consuming problem of inverse kinematics of robot arm, a closed inverse kinematics solution algorithm for humanoid dual-arm robot was proposed. The effectiveness of the algorithm was verified by simulation.
\end{abstract}

(C) 2021 The Authors. Published by Atlantis Press B.V.

This is an open access article distributed under the CC BY-NC 4.0 license (http://creativecommons.org/licenses/by-nc/4.0/).

\section{INTRODUCTION}

Humanoid dual-arm robot is a kind of bionic robot designed by imitating the shape, structure and function of human body. It can work with both hands cooperatively just like human. The inverse kinematics of manipulator mainly includes geometric method, analytical method and numerical method. The geometric method is a special case of analytic method in some cases, and its applicability is weak $[1,2]$. Analytical inverse kinematics of the manipulator can efficiently obtain all the inverse solutions of the manipulator in the desired position, but the manipulator must satisfy the Piper criterion [3]. The numerical method has no special requirements for the joint number and structure of the manipulator, but it needs to be solved through continuous iteration, which not only takes a long time, but the average calculation error is also 10 times that of the analytical method $[4,5]$.

Because it takes a long time to meet the accuracy requirement and solve the problem of manipulator inverse kinematics solution, the analytical solution is adopted. In Section 2, a closed inverse kinematics algorithm is proposed for the humanoid robot arms. The algorithm is used for simulation analysis in Section 3. And we verify the effectiveness of the algorithm in Section 4.

\section{KINEMATIC MODELING OF DUAL-ARM ROBOT}

\subsection{Forward Kinematics}

The robot model in this paper is shown in Figure 1. Each arm of the robot has six Degrees of Freedom (DOF). The three axes

“Corresponding author. Email: 19121251@bjtu.edu.cn of the shoulder joint intersect at one point. Because this case conforms to the Piper criterion, there are analytic solutions. In general, the end of the robot meets the Piper criterion, but the shoulder joint of the humanoid robot generally meets the Piper criterion. Although it looks the same, their solutions are different. Taking the shoulder joint as the basic coordinate frame [6], Figure 1 also shows the link coordinate frames of the right arm and its $D-H$ parameters. Where joint $i$ represents the $i$-th joint of the manipulator, $\theta_{i}$ is the angle between $x_{i-1}$ and $x_{i}, d_{i}$ is the distance between $x_{i-1}$ and $x_{i}, a_{i}$ is the distance between $z_{i-1}$ and $z_{i}$, $\alpha_{i}$ is the angle between $z_{i-1}$ and $z_{i}$. The left arm and the right arm are identical.

The position and orientation of the end-effector can be obtained by chain-multiplying the six link-transformation matrices together to obtain the spatial displacement of the sixth coordinate frame with respect to the base coordinate frame:

$$
\begin{aligned}
{ }^{0} T_{6} & =\prod_{i=1}^{6}{ }^{i-1} A_{i}={ }^{0} A_{1}{ }^{1} A_{2}{ }^{2} A_{3}{ }^{3} A_{4}{ }^{4} A_{5}{ }^{5} A_{6} \\
& =\left[\begin{array}{llll}
x_{6} & y_{6} & z_{6} & p_{6} \\
0 & 0 & 0 & 1
\end{array}\right]=\left[\begin{array}{llll}
n & s & a & p \\
0 & 0 & 0 & 1
\end{array}\right]
\end{aligned}
$$

Where $x_{i}, y_{i}$ and $z_{i}$ represent the unit vectors along the principal axes of the coordinate frame $i,{ }^{i-1} A_{i}$ is a general link transformation matrix, relating the $i$-th coordinate frame to the $(i-1)$ th coordinate frame, and $[n, s, a, p]$ represents the normal vector, the sliding vector, the approach vector and the position vector of the hand respectively.

\subsection{Inverse Kinematics}

We take the inverse of both sides of Equation (1). The new matrix $T^{\prime}$ is 


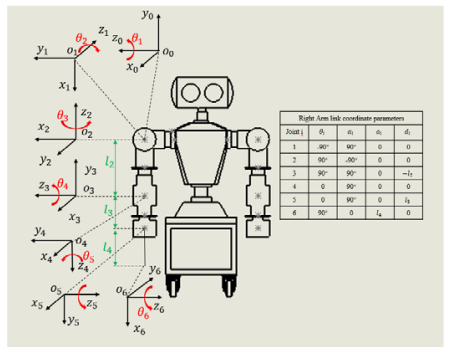

Figure 1 Link coordinate frames of the right arm and its $D-H$ parameters.

$$
\begin{aligned}
T^{\prime} & =\left[\begin{array}{cccc}
n & s & a & p \\
0 & 0 & 0 & 1
\end{array}\right]^{-1}=\left[\begin{array}{cccc}
n^{\prime} & s^{\prime} & a^{\prime} & p^{\prime} \\
0 & 0 & 0 & 1
\end{array}\right] \\
& ={ }^{6} A_{5}^{5} A_{4}^{4} A_{3}^{3} A_{2}^{2} A_{1}^{1} A_{0}={ }^{6} A_{0}
\end{aligned}
$$

We can obtain an equation that we label as $E_{2}$ equation by moving the link transformation matrix ${ }^{5} A_{6}$ to the left-hand side of Equation (2).

$$
{ }^{5} A_{6} T^{\prime}={ }^{5} A_{4}{ }^{4} A_{3}{ }^{3} A_{2}{ }^{2} A_{1}^{1} A_{0}
$$

The left-hand side of $E_{2}$ is

$$
E_{L 2}=\left[\begin{array}{cccc}
\cdots & \cdots & \cdots & C_{6}\left(p_{x}^{\prime}+l_{L 4}\right)-S_{6} p_{y}^{\prime} \\
\cdots & \cdots & \cdots & S_{6}\left(p_{x}^{\prime}+l_{L 4}\right)+C_{6} p_{y}^{\prime} \\
\cdots & \cdots & \cdots & p_{z}^{\prime} \\
0 & 0 & 0 & 1
\end{array}\right]
$$

And the right-hand side of $E_{2}$ is

$$
E_{R 2}=\left[\begin{array}{cccc}
\cdots & \cdots & \cdots & S_{4} C_{5} l_{L 2} \\
\cdots & \cdots & \cdots & -C_{4} l_{L 2}-l_{L 3} \\
\cdots & \cdots & \cdots & S_{4} S_{5} l_{L 2} \\
0 & 0 & 0 & 1
\end{array}\right]
$$

Where $S_{i} \equiv \sin \theta_{i}, C_{i} \equiv \cos \theta_{i}, S_{i j} \equiv \sin \left(\theta_{i}+\theta_{j}\right), C_{i} \equiv \cos \left(\theta_{i}+\theta_{j}\right)$, and $l_{L i}$ are geometric link parameters in Figure 1 . By comparing the elements $(1,4),(2,4)$ and $(3,4)$ of the $E_{L 2}$ and $E_{R 2}$, we can obtain $C_{4}$ and then $S_{4}$ from $C_{4}$, and from which we can find the joint solution for $\theta_{4}$. we can solve $\theta_{5}$ when we have solved $\theta_{4}$. Similarly, we can solve $\theta_{6}$ when we have solved $\theta_{4}$ and $\theta_{5}$.

$$
\begin{aligned}
& C_{4}=\frac{\left(p_{x}^{\prime}+l_{L 4}\right)^{2}+p_{y}^{\prime 2}+p_{z}^{\prime 2}-l_{L 2}^{2}-l_{L 3}^{2}}{2 l_{L 2} l_{L 3}} \\
& S_{4}= \pm \sqrt{1-C_{4}^{2}} \\
& \theta_{4}=a \tan 2\left(S_{4}, C_{4}\right) \\
& S_{5}=\frac{p_{z}^{\prime}}{\left(S_{4} l_{L 2}\right)} \\
& C_{5}= \pm \sqrt{1-S_{5}^{2}} \\
& \theta_{5}=a \tan 2\left(S_{5}, C_{5}\right) \\
& \theta_{6}=a \tan 2\left(-\left(C_{4} l_{L 2}+l_{L 3}\right), S_{4} C_{5} l_{L 2}\right)-a \tan 2\left(p_{y}^{\prime}, p_{x}^{\prime}+l_{L 4}\right)
\end{aligned}
$$

To solve for the remaining joint angles, we can move the link transformation matrix ${ }^{3} A_{4}{ }^{4} A_{5}$ to the left-hand side of Equation (3). And we can obtain an equation that we label as $E_{4}$ equation.

$$
{ }^{3} A_{4}{ }^{4} A_{5}{ }^{5} A_{6} T^{\prime}={ }^{3} A_{2}{ }^{2} A_{1}{ }^{1} A_{0}
$$

The left-hand side of $E_{4}$ is

$$
E_{L 4}=\left[\begin{array}{cccc}
a 11 & a 12 & a 13 & a 14 \\
a 21 & a 22 & a 23 & a 24 \\
a 31 & a 32 & a 33 & a 34 \\
0 & 0 & 0 & 1
\end{array}\right]
$$

And the right-hand side of $E_{4}$ is

$$
E_{R 4}=\left[\begin{array}{cccc}
C_{1} C_{2} C_{3}-S_{1} S_{3} & C_{1} S_{3}+S_{1} C_{2} S_{3} & S_{2} C_{3} & 0 \\
-C_{1} S_{2} & -S_{1} S_{2} & C_{2} & l_{L 2} \\
S_{1} C_{3}+C_{1} C_{2} S_{3} & S_{1} C_{2} S_{3}-C_{1} C_{3} & S_{2} S_{3} & 0 \\
0 & 0 & 0 & 1
\end{array}\right]
$$

By comparing the element $(2,3)$ of $E_{L 4}$ and $E_{R 4}$, we get $C_{2}$ and then $S_{2}$, and the joint solution $\theta_{2}$ from them,

$$
\begin{aligned}
C_{2} & =a 23=a_{z}^{\prime} S_{4} S_{5}-a_{y}^{\prime}\left(C_{4} C_{6}+S_{4} C_{5} S_{6}\right)-a_{x}^{\prime}\left(C_{4} S_{6}+S_{4} C_{5} C_{6}\right) \\
S_{2} & = \pm \sqrt{1-C_{2}^{2}} \\
\theta_{2} & =a \tan 2\left(S_{2}, C_{2}\right)
\end{aligned}
$$

By comparing the elements $[(1,3)(3,3)]$ and $[(2,1)(2,2)]$, we can find the joint solution $\theta_{3}$ and $\theta_{1}$,

$$
\begin{aligned}
a 13 & =a_{x}^{\prime}\left(C_{4} C_{5} C_{6}+S_{4} S_{6}\right)+C_{4} S_{5} a_{z}^{\prime}+\left(S_{4} C_{6}-C_{4} C_{5} S_{6}\right) a_{y}^{\prime} \\
a 33 & =S_{5} C_{6} a_{x}^{\prime}-C_{5} a_{z}^{\prime}-S_{5} S_{6} a_{y}^{\prime} \\
\theta_{3} & =a \tan 2(a 33, a 13) \\
a 21 & =\left(S_{4} C_{5} C_{6}-C_{4} S_{6}\right) n_{x}^{\prime}+S_{4} S_{5} n_{z}^{\prime}-\left(S_{4} C_{5} S_{6}+C_{4} C_{6}\right) n_{y}^{\prime} \\
a 22 & =\left(S_{4} C_{5} C_{6}-C_{4} S_{6}\right) s_{x}^{\prime}+S_{4} S_{5} s_{z}^{\prime}-\left(S_{4} C_{5} S_{6}+C_{4} C_{6}\right) s_{y}^{\prime} \\
\theta_{1} & =a \tan 2(-a 22,-a 21)
\end{aligned}
$$

The solution of the six joints is obtained by the above procedure. The left arm is exactly the same as the right arm for the solution.

\section{DUAL-ARM ROBOT MODELING BASED ON SIMSCAPE}

\subsection{Model Building}

In this paper, the dual-arm robot simulation model mainly includes three parts, namely the trajectory planning joint angle input module, the robot body module and the output measurement module. Its Simscape Multibody model is shown in Figure 2.

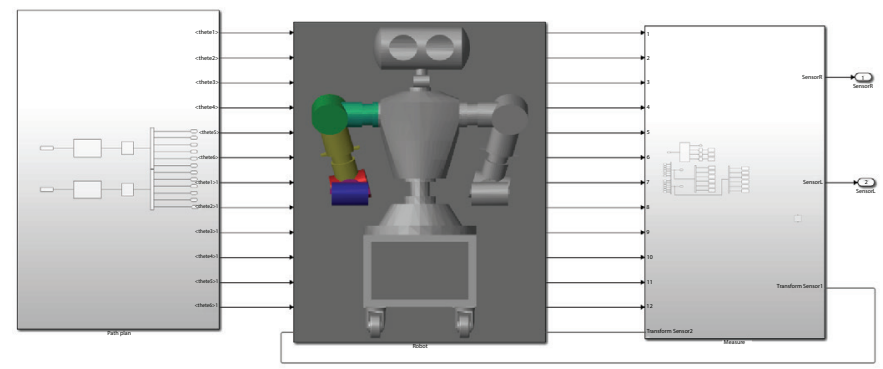

Figure 2 Dual-arm robot simulation model. 


\subsection{Establishment of Joint Angle Input Module for Trajectory Planning}

We plan the trajectory of space in Cartesian coordinates. After solving the inverse kinematics function, we can obtain the angles of each joint. Then we output these angles to the various joints of the arm. The joint angle input model for trajectory planning is shown in Figure 3.

\subsection{Establishment of Robot Ontology Module}

Figure 4 shows the robot ontology module. It includes world coordinate frame and robot ontology. The robot has two arms. Each arm has six DOF, including three DOF for shoulder joint, one DOF for elbow joint and two DOF for wrist joint.

\subsection{Establishment of Output Measurement Module}

The output measurement module shown in Figure 5 can measure the rotation angle of each DOF. Through this module, we can also obtain the displacement of the terminal coordinate frame of the manipulator relative to the base coordinate frame.

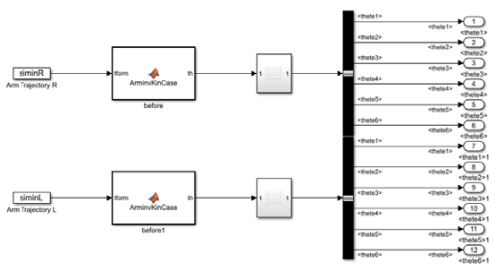

Figure 3 The joint angle input model for trajectory.

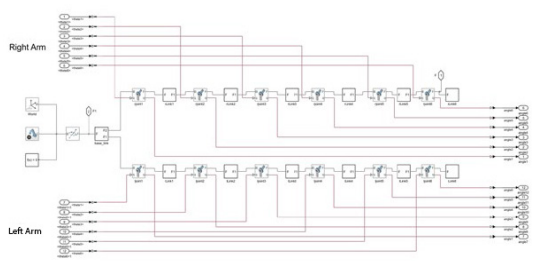

Figure 4 The robot ontology module.

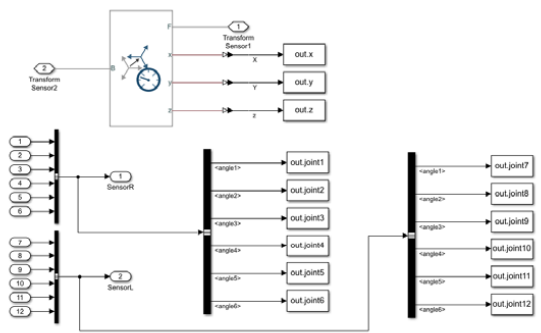

Figure 5 The output measurement module.
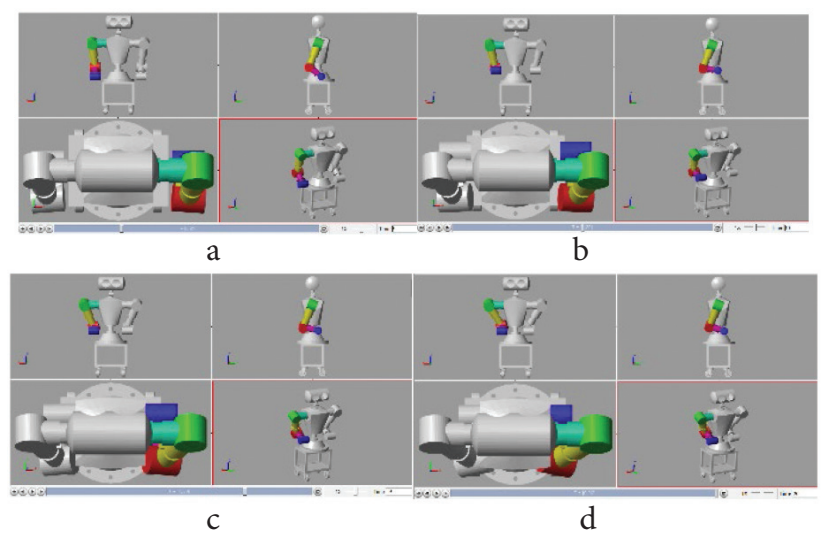

Figure 6 Simulation diagram of each moment. (a) $t=5 \mathrm{~s}$. (b) $t=10 \mathrm{~s}$. (c) $t=15 \mathrm{~s}$. (d) $t=20 \mathrm{~s}$.
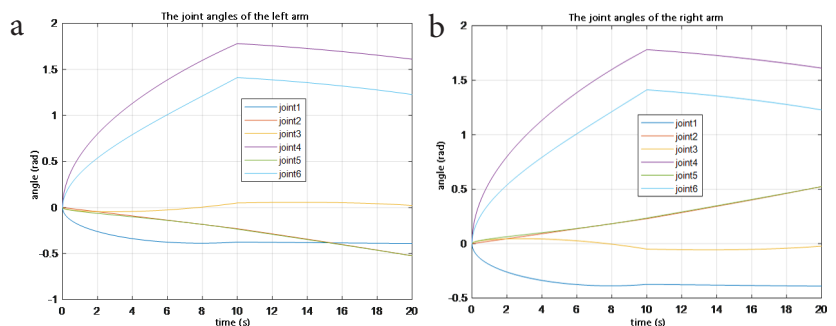

Figure 7 The motion angles of each joint of the (a) left and (b) right arms.

\section{SIMULATION VERIFICATION AND ANALYSIS}

\subsection{Simulation Process}

The robot first raised their arms and then approached each other The entire process is shown in Figure 6.

\subsection{The Motion Angles of Each Joint}

Figure 7 shows the motion angles of each joint of the manipulator. At the initial moment, the angle of each joint is 0 radian, and at about $10 \mathrm{~s}$, the robotic arms begin to approach each other. The angle of each joint is continuous in the whole process, which proves that the inverse kinematics solution algorithm in this paper can solve the analytic solution effectively.

\section{CONCLUSION}

In this paper, the kinematics modeling of dual-arm robot is carried out. We present an analytical solution method for six DOF dual-arm robot for each arm. Simulation results show that the proposed method is effective. In the future, it will be applied to the arm trajectory planning of the robot.

\section{CONFLICTS OF INTEREST}

The authors declare they have no conflicts of interest. 


\section{ACKNOWLEDGMENT}

This work was supported by the Key Research and Development Program (No. M18GY300021).

\section{REFERENCES}

[1] I.S. Fischer, A geometric method for determining joint rotations in the inverse kinematics of robotic manipulators. J. Robot. Syst. 17 (2000), 107-117.

[2] S. Yahya, M. Moghavvemi, H.A.F. Mohamed, Geometrical approach of planar hyper-redundant manipulators: inverse kinematics, path planning and workspace, Simul. Modell. Pract. Theory 19 (2011), 406-422.
[3] S.R. Buss, Introduction to inverse kinematics with Jacobian transpose, pseudoinverse and damped least squares methods, J. IEEE Trans. Robot. Autom. 16 (2004), 1-19.

[4] Z. Sui, L. Jiang, Y.T. Tian, W. Jiang, Genetic algorithm for solving the inverse kinematics problem for general 6R robots, in: Z. Deng, H. Li (Eds.), Proceedings of the 2015 Chinese Intelligent Automation Conference, Springer, Berlin, Heidelberg, 2015, pp. 151-161.

[5] M. Bayati, Using cuckoo optimization algorithm and imperialist competitive algorithm to solve inverse kinematics problem for numerical control of robotic manipulators, J. Syst. Control Eng. 229 (2015), 375-387.

[6] M.A. Ali, H. Andy Park, C.S. George Lee, Closed-form inverse kinematic joint solution for humanoid robots, 2010 IEEE/RSJ International Conference on Intelligent Robots and Systems, IEEE, Taipei, Taiwan, 2010, pp. 704-709.

\section{AUTHORS INTRODUCTION}

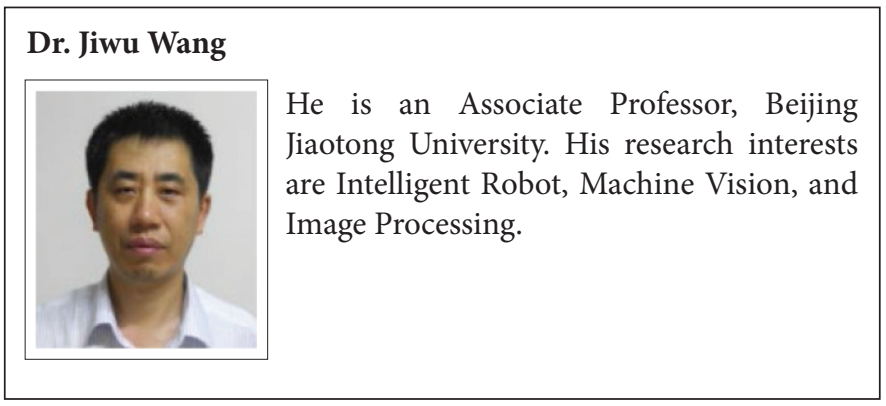

\section{Mr. Junxiang Xu}

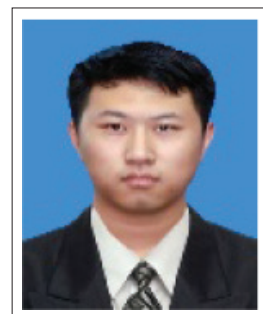

$\mathrm{He}$ is a postgraduate in Beijing Jiaotong University. His research interest is Robot Modeling and Simulation. 